\title{
On the modified Reynolds equation for journal bearings in a case of non-Newtonian Rabinowitsch fluid model
}

\author{
Juliana Javorova ${ }^{1, *}$, and Jordanka Angelova ${ }^{2}$ \\ ${ }^{1}$ UCTM, Dept. of Applied Mechanics, 8 Kliment Ohridski Blvd., 1756 Sofia, Bulgaria \\ ${ }^{2}$ UCTM, Dept. of Mathematics, 8 Kliment Ohridski Blvd., 1756 Sofia, Bulgaria
}

\begin{abstract}
In this paper, a theoretical analysis of hydrodynamic plain journal bearings with finite length at taking into account the effect of nonNewtonian lubricants is presented. Based upon the Rabinowitsch fluid model (cubic stress constitutive equation) and by integrating the continuity equation across the film, the nonlinear modified 2D Reynolds type equation is derived in details so that to study the dilatant and pseudoplastic nature of the lubricant in comparison with Newtonian fluid. A dimensionless equation of hydrodynamic pressure distribution in a form appropriate for numerical modeling is also presented. Some particular cases of $1 \mathrm{D}$ applications can be recovered from the present derivation.
\end{abstract}

\section{Introduction}

Hydrodynamic journal bearings are considered to be a vital component of all rotating machines. Journal bearings are used widely for thousands of years in many areas of mechanical engineering. Currently large numbers of them are applied successfully in machine tools, automotive and aircraft piston engines, turbo-machinery, micro-electromechanical systems, etc. Furthermore, it is well known that the performance of bearings is strongly influenced by the composition and rheological characteristics of the lubricant.

In order to meet requirements of the modern machinery systems, it is necessary to look for the enhancement in lubricating performance of all kind of bearings and other types of lubricated contacts. In this relation, currently, the use of Newtonian fluids blended with various additives increases, which is based on the effective improvement in the bearing characteristics as compared to the lubrication with Newtonian lubricants. These additives often are viscosity index improvers which represent high molecular weight polymers such as polyisobutylene, polymethacrylate, ethylene propylene, etc. These kinds of lubricants exhibit pseudoplastic and dilatant behaviour of non-Newtonian lubricants, in which the ratio between the shear stress and shear rate is no longer a constant. According to the experimental work of Wada and Hayashi [1] the non-Newtonian rheological behaviour of such kinds of lubricants with additives can be represented by an empiric cubic stress model, also called Rabinowitsch fluid model.

\footnotetext{
*Corresponding author: july@uctm.edu
} 
Applying this fluid model, a number of scientific papers concerning the subjects of nonNewtonian fluid film lubrication have been published. For instance, the theoretical study of bearing performance with non-Newtonian lubricants using Rabinowitsch fluid model is done on journal bearings by Wada and Hayashi [1], Rajalingham et al. [2], Bourgin and Gay [3], Sharma et al. [4], Javorova et al. [5, 6], Kushare [7], Lin et al. [8]. Some contributions for squeeze film bearings are presented by Hashimoto and Wada [9], Lin [10], Naduvamani [11], Shigehalli [12]. Furthermore, Lin et al. [13-15] and Singh et al. [16-20] used the model of Rabinowitsch fluid to investigate in details the performance of different types of hydrostatic, hydrodynamic and squeeze film bearing systems. More general lubrication problems for the hybrid bearings can be found in the works of Walicka et al. [21, 22], Ratajczak et al. [23] as the same authors work also on trust bearings [24]. Recently several papers in the field of peristaltic flow in a tube with Rabinowitsch fluid model are also published.

As a whole, according to results in the mentioned studies, the influences of nonNewtonian Rabinowitsch fluids on the lubrication performances of journal bearings, slider bearings, squeeze film bearings, hydrostatic bearings, and other types of lubricated contacts are significantly apparent. It is shown that the steady state and squeeze-film performances are strongly affected by non-Newtonian effects based on the Rabinowitsch fluid model.

The most of above mentioned papers aimed to obtain some modified Reynolds equation for such kind of lubricants as well as to receive an analytical solution of it for more simple cases. For more complicated ones, as behaviour of finite length journal bearings, the authors regularly apply modified Reynolds equation obtained by Wada and Hayashi derived in short in [1]. In the mentioned studies a detailed mathematical output of modified Reynolds equation is not given that motivates authors to present here such one. The current study aims to represent consistently and in details a mathematical procedure to obtain the nonlinear modified two-dimensional Reynolds type equation based on the Rabinowitsch fluid model. By this way it is possible to study the pseudoplastic and dilatant nature of lubricants with long chain polymer additives in comparison with Newtonian fluids.

\section{Modeling of the hydrodynamic lubrication in journal bearings}

A radial journal bearing with finite length is considered under steady state conditions. The lubricant fluid in bearing clearance has non-Newtonian properties. The rheological fluid law described by the Rabinowitsch model [1, 13, 18, etc.] is presented with the following cubic equation

$$
\tau+k \tau^{3}=\eta \frac{d u}{d y}
$$

where: $\tau$ - shear stress in the fluid film; $k$ - coefficient of pseudoplasticity (parameter responsible for the lubricants non-Newtonian behaviour); $\eta$ - initial viscosity of the lubricant; $u, v, w$ - velocity components in the directions of Cartesian coordinates $x, y, z$, respectively.

In dependence of the values of the coefficient of pseudoplasticity $k$ there are three different groups of lubricants: for $k>0$ - pseudoplastic fluids, if $k=0$ - Newtonian fluid, as for $k<0$ - dilatant fluid. 

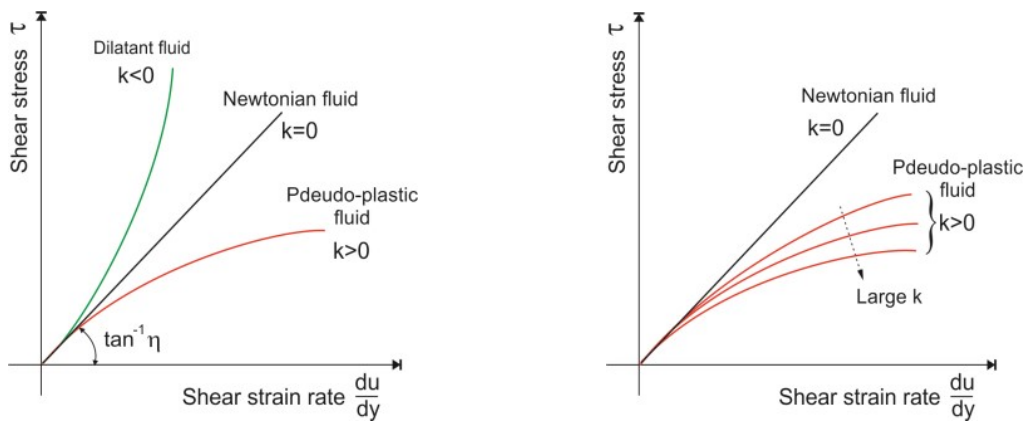

Fig. 1. Flow curves for non-Newtonian Rabinowitsch fluid.

The flow characteristics of these kinds of lubricants are shown in Fig. 1. Here $\eta$ is a tangent at the original point of flow curves because of which it is called "initial viscosity" by analogy with $[1,13,17]$. If values of $\eta$ do not vary, the nonlinearity of the flow curve increases with the value of the coefficient of pseudoplasticity $k$ (see Fig. 1 - on the right).

\subsection{Bearing geometry}

For the geometry of the considered plane journal bearing with $360^{\circ}$ range are introduced the following assumptions (see Fig.2): The journal and the bearing have a round shape and parallel axes; their surfaces are perfectly smooth. The bearing gap is filled with a lubricating fluid with a constant pressure equal to the external. The journal rotates with a constant angular velocity $\omega$ around its axis. The bearing radius $R$ is approximately equal to the journal radius $r(R \approx r)$. The radial bearing clearance $c=R-r$ is of the order $1.10^{-4}$, i.e. $c<<1$, see Fig. 1 . Therefore the clearance ratio $\beta=c / r \approx 1.10^{-3}<<1$. The bearing eccentricity is also very small, so the eccentricity ratio $\varepsilon=e / r \approx 1.10^{-3}<<1$. Moreover, for all types of bearings the fluid film thickness $h$ is very small compared to the other dimensions of the contact surfaces because of which the ratio $h / l \approx h / r \approx 1.10^{-3}<<1$, where $l$ is the fluid film length in a circumferential direction. All of the above mentioned about the bearing geometry justifies the commonly accepted hypothesis in the theory of hydrodynamic lubrication to neglect the curvature of the lubricated surfaces and, respectively, the curvature of the lubricant film.
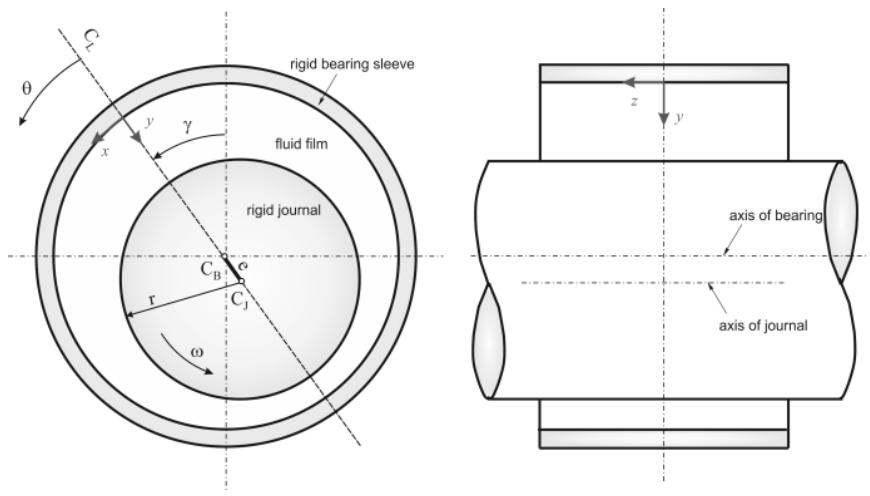

Fig. 2. Hydrodynamic journal bearing. 


\subsection{Boundary value problem}

The constitutive equations between the shear stress and rate of share strain for two dimensional flow of the Rabinowitsch fluid obeys the following nonlinear relationships

$$
\begin{aligned}
& \tau_{x y}+k \tau_{x y}^{3}=\eta \frac{d u}{d y}, \\
& \tau_{z y}+k \tau_{z y}^{3}=\eta \frac{d w}{d y},
\end{aligned}
$$

where $\tau_{x y}$ and $\tau_{z y}$ are shear stress components in $x$ and $z$ directions, respectively.

According to the thin film theory of hydrodynamic lubrication [25, 1, 18], the momentum and continuity equations in Cartesian coordinates are represented by the following differential equations:

$$
\begin{gathered}
\frac{\partial p}{\partial x}=\frac{\partial \tau_{x y}}{\partial y} ; \\
\frac{\partial p}{\partial y}=0 \\
\frac{\partial p}{\partial z}=\frac{\partial \tau_{z y}}{\partial y} ; \\
\frac{\partial u}{\partial x}+\frac{\partial v}{\partial y}+\frac{\partial w}{\partial z}=0
\end{gathered}
$$

where $p$ is the hydrodynamic pressure.

Related to the bearing configuration, the boundary conditions for the fluid velocity components are

at $y=0$ :

$$
u(x, 0, z)=u_{0}=0 ; \quad v(x, 0, z)=v_{0}=0 ; \quad w(x, 0, z)=w_{0}=0,
$$

at $y=h$ :

$$
u(x, h, z)=u_{h}=U=\omega r ; \quad v(x, h, z)=v_{h}=\omega r \frac{\partial h}{\partial x} ; \quad w(x, h, z)=w_{h}=0,
$$

where: $\omega$ is the journal angular velocity, $h=h(x, z)$ is the fluid film thickness.

\subsection{Mathematical procedure}

The integration of (3.a) and (3.c) with respect to $y$ yields:

$$
\begin{gathered}
\tau_{x y}=\frac{\partial p}{\partial x} y+\tau_{1} \\
\tau_{z y}=\frac{\partial p}{\partial z} y+\tau_{2},
\end{gathered}
$$


where $\tau_{1}$ and $\tau_{2}$ are integration constants.

By substitution of (6.a) and (6.b) in (2.a) and (2.b), respectively, it is obtained:

$$
\frac{d f}{d y}=\frac{1}{\eta}\left[q y+\xi+k(q y+\xi)^{3}\right]
$$

where $f \in\{u, w\}, q \in\{\partial p / \partial x, \partial p / \partial z\}, \xi \in\left\{\tau_{1}, \tau_{2}\right\}$. Here the couples $\{. . . .$.$\} are ordered and$ mapping between them one-to-one.

Integration of (7) with respect to $y \in[0, h]$ leads to

$$
\eta[f(h)-f(0)]=\int_{0}^{h}\left[q y+\xi+k(q y+\xi)^{3}\right] d y
$$

where: $f(h) \in\left\{u_{h}, w_{h}\right\}, f(0) \in\left\{u_{0}, w_{0}\right\}$.

Applying boundary conditions (4) and (5) and performing some elementary transformations the following cubic equation for $\xi$ is obtained:

$$
\xi^{3}+\frac{3}{2} q h \xi^{2}+\left[(q h)^{2}+\frac{1}{k}\right] \xi+\frac{(q h)^{3}}{4}+\frac{q h}{2 k}-\frac{\eta f(h)}{h k}=0 .
$$

On the other hand, reordering (9) in ascending order by power of $\zeta=\xi+\frac{q h}{2}$ leads to a semi-cubic equation of $\zeta$ :

$$
\zeta^{3}+\left[\frac{1}{k}+\left(\frac{q h}{2}\right)^{2}\right] \zeta-\frac{\eta f(h)}{h k}=0 .
$$

If $f(h)=0 /$ see (4) for $w_{h} /$, then $\zeta=\xi+\frac{q h}{2}=0$, and $\xi=-\frac{q h}{2}$, i.e. $\tau_{2}=-\frac{q h}{2}$.

According to [1], since the value of $\lambda=\left|\frac{\eta u_{h}}{h\left[1+k(q h / 2)^{2}\right]}\right|$ is enough small within the range of parameters considered, then from (8) follows that $\tau_{1}=-\frac{q h}{2}+\lambda, \quad \lambda^{2} \approx \lambda^{3} \approx 0$.

Furthermore, by integrating (7) with respect to $s \in[0, y]$ it is possible to find an antiderivative of $f$ as

$$
f(y)-f(0)=\frac{1}{\eta}\left[\int_{0}^{y}(q s+\xi) d s+k \int_{0}^{y}(q s+\xi)^{3} d s\right],
$$

where $s$ - variable of integration.

Afterwards, based on (11) the velocity component $u$ is obtained in a form: 


$$
\begin{gathered}
u=\frac{1}{\eta}\left[q \int_{0}^{y}\left(s-\frac{h}{2}\right) d s+\lambda y+k \int_{0}^{y}\left(q s-\frac{q h}{2}+\lambda\right)^{3} d s\right]= \\
=\frac{1}{\eta}\left\{q \Phi_{1}(y)+\lambda y+k\left[q^{3} \int_{0}^{y}\left(s-\frac{h}{2}\right)^{3} d s+3 q^{2} \lambda \int_{0}^{y}\left(s-\frac{h}{2}\right)^{2} d s+3 q \lambda^{2} \int_{0}^{y}\left(s-\frac{h}{2}\right) d s+\lambda^{3} y\right]\right\} .
\end{gathered}
$$

Neglecting in (12) terms multiplying by second and third powers of $\lambda$ yields

$$
u \approx \frac{1}{\eta}\left[q \Phi_{1}(y)+k q^{3} \Phi_{2}(y)\right]+\frac{\lambda\left[y+k q^{2} \Phi_{3}(y)\right]}{\eta},
$$

where:

$$
\begin{gathered}
\Phi_{1}(y)=\int_{0}^{y}\left(s-\frac{h}{2}\right) d s=\frac{1}{2} y(y-h) ; \\
\Phi_{2}(y)=\int_{0}^{y}\left(s-\frac{h}{2}\right)^{3} d s=\frac{1}{4}\left(2 y^{2}-2 h y+h^{2}\right) \Phi_{1}(y) ; \\
\Phi_{3}(y)=3 \int_{0}^{y}\left(s-\frac{h}{2}\right)^{2} d s=\frac{1}{4} y\left(4 y^{2}-3 h y+3 h^{2}\right) .
\end{gathered}
$$

The expression for $w$ is similar to (13) without the term multiply by $\lambda$. Therefore

$$
\begin{gathered}
u \approx \frac{1}{\eta}\left[q \Phi_{1}(y)+k q^{3} \Phi_{2}(y)\right]+\frac{u_{h}\left[y+k q^{2} \Phi_{3}(y)\right]}{h\left[1+k(q / 2)^{2} h^{2}\right]}, \quad q=\frac{\partial p}{\partial x} \\
w=\frac{1}{\eta}\left[q \Phi_{1}(y)+k q^{3} \Phi_{2}(y)\right], \quad q=\frac{\partial p}{\partial z} .
\end{gathered}
$$

Integrating the continuity equation (3.d) with respect to $y \in[0, h]$ leads to

$$
\int_{0}^{h} \frac{\partial u}{\partial x} d y+\int_{0}^{h} \frac{\partial v}{\partial y} d y+\int_{0}^{h} \frac{\partial w}{\partial z} d y=0
$$

As the lubricant film thickness $h=h(x, z)$, using the Leibniz integral rule

$$
\frac{\partial}{\partial s} \int_{a(s)}^{b(s)} f(x, s) d x=\int_{a(s)}^{b(s)} \frac{\partial f(x, s)}{\partial s} d x+f(b(s), s) b^{\prime}(s)-f(a(s), s) a^{\prime}(s)
$$

and applying the boundary conditions, for the continuity equation (17) follows

$$
\frac{\partial}{\partial x} \int_{0}^{h} u d y-u_{h} \frac{\partial h}{\partial x}+v_{h}+\frac{\partial}{\partial z} \int_{o}^{h} w d y=0 .
$$


Integrating the first term of (18) with respect to $y \in[0, h]$ and rendering into account (15) leads to

$$
\int_{0}^{h} u d y=\frac{1}{\eta}\left[q \int_{0}^{h} \Phi_{1}(y) d y+k q^{3} \int_{0}^{h} \Phi_{2}(y) d y\right]+\frac{\lambda}{\eta}\left[\int_{0}^{h} y d y+k q^{2} \int_{0}^{h} \Phi_{3}(y) d y\right] .
$$

Analogously, the integral of $w$ in the forth term of (18) is obtained by (16) in a form

$$
\int_{0}^{h} w d y=\frac{1}{\eta}\left[q \int_{0}^{h} \Phi_{1}(y) d y+k q^{3} \int_{0}^{h} \Phi_{2}(y) d y\right] .
$$

By using (14) the integrals of $\Phi_{1}, \Phi_{2}, \Phi_{3}$ in (19) and (20) are respectively equal to

$$
\int_{0}^{h} \Phi_{1}(y) d y=-\frac{h^{3}}{12} ; \quad \int_{0}^{h} \Phi_{2}(y) d y=-\frac{h^{5}}{80} ; \quad \int_{0}^{h} \Phi_{3}(y) d y=\frac{h^{4}}{8} .
$$

Then after substitution of the velocity components (15), (16) in (18) and using (21) the following Reynolds type equation is obtained

$$
\frac{1}{\eta} \frac{\partial}{\partial x}\left[\frac{h^{3}}{12} \frac{\partial p}{\partial x}+k \frac{h^{5}}{80}\left(\frac{\partial p}{\partial x}\right)^{3}\right]+\frac{1}{\eta} \frac{\partial}{\partial z}\left[\frac{h^{3}}{12} \frac{\partial p}{\partial z}+k \frac{h^{5}}{80}\left(\frac{\partial p}{\partial z}\right)^{3}\right]=\frac{\omega r}{2} \frac{\partial h}{\partial x} .
$$

This is the modified Reynolds equation for a finite length journal bearing lubricated with non-Newtonian Rabinowitsch fluid under steady state conditions. The type of equation coincides with the one obtained in [1] as here the coefficient of pseudoplasticity $k$ is also involved. It is known that for Newtonian fluids $k=0$ and from (22) can be obtained the classical Reynolds equation for Newtonian fluids. The above equation is more general than classical one, as it includes the case of non-Newtonian lubricants $(k \neq 0)$.

This equation has to be represented in a non-dimensional form by introducing the coordinate transformations and corresponding dimensionless variables, according to substitutions:

$$
\theta=\frac{x}{R} \approx \frac{x}{r} ; \quad z_{1}=\frac{z}{L / 2} ; \quad \alpha=\frac{2 r}{L} ; \quad \beta=\frac{c}{r} ; \quad \Pi=\frac{c^{2}}{6 \eta U r} p ; \quad H=\frac{h}{c},
$$

where: $\theta$ - circumferential coordinate, $z_{1}$ - dimensionless axial coordinate, $L$ - bearing axial length, $\alpha$ - diameter to length ratio, $\beta$ - clearance ratio, $\Pi$ - dimensionless pressure, $U=\omega r$ - journal circumferential velocity, $H$ - dimensionless film thickness.

All variables in (22) are changed with non-dimensional ones (23), as for the partial derivatives the following equalities are in effect

$$
\begin{array}{ll}
\frac{\partial}{\partial x}=\frac{1}{r} \frac{\partial}{\partial \theta} ; & \frac{\partial p}{\partial x}=\frac{\partial \Pi}{\partial \theta} \frac{6 \eta \omega r}{c^{2}} ; \\
\frac{\partial}{\partial z}=\frac{1}{L / 2} \frac{\partial}{\partial z_{1}} ; & \frac{\partial p}{\partial z}=\frac{\partial \Pi}{\partial z_{1}} \frac{6 \eta \omega r^{2}}{(L / 2) c^{2}}
\end{array}
$$




$$
\frac{\partial h}{\partial x}=\frac{c}{r} \frac{\partial H}{\partial \theta}
$$

After substitution of (23) and (24) in (22) and applying some mathematical transformations the final dimensionless form of $2 \mathrm{D}$ Reynolds equation for pressure distribution is obtained as:

$$
\frac{\partial}{\partial \theta}\left[\frac{H^{3}}{12} \frac{\partial \Pi}{\partial \theta}+\psi \frac{36 H^{5}}{80}\left(\frac{\partial \Pi}{\partial \theta}\right)^{3}\right]+\alpha^{2} \frac{\partial}{\partial z_{1}}\left[\frac{H^{3}}{12} \frac{\partial \Pi}{\partial z_{1}}+\alpha^{2} \psi \frac{36 H^{5}}{80}\left(\frac{\partial \Pi}{\partial z_{1}}\right)^{3}\right]=\frac{1}{12} \frac{\partial H}{\partial \theta} .
$$

In this equation the non-dimensional nonlinear factor $\psi$, which takes into account the non-Newtonian behaviour of a fluid, described by the cubic model, is involved. According to $[1,13,18$, etc. $]$ the nonlinear factor is represented by the following relationship

$$
\psi=k\left(\frac{\eta U}{c}\right)^{2}=k\left(\frac{\eta \omega r}{c}\right)^{2}
$$

When the nonlinear factor $\psi$ is set to zero, the Reynolds classical equation for a journal bearing lubricated with a Newtonian fluid is obtained. At pseudoplastic non-Newtonian fluids $\psi>0$, while at the dilatant ones $\psi<0[13,26,18]$. As it can be seen from (26), the nonlinear factor values depend on the coefficient of pseudoplasticity, the lubricant initial viscosity, the velocity of journal, and the radial clearance. Given the relation (26) between the nonlinear factor and the coefficient of pseudoplasticity, the increase of $k$ increases the values of $\psi$. For Newtonian fluids $\psi=0$ because $k=0$. Namely by introducing this dimensionless variable into equation (25), the rheological characteristics of the pseudoplastic and dilatant fluids, which can be described by the Rabinowitsch fluid model, are taken into consideration in the mathematical model.

\section{Particular cases}

As it was written in [1], the analysis of Reynolds type equation as (23) can be performed for small and respectively for large values of the nonlinear factor by using different approaches.

Furthermore, for small nonlinear factor two particular cases of 1D journal bearings lubricated with a Rabinowitsch fluid can be considered at using the long and short bearing approximations, which are by the other side extensively applied in the studies of lubrication theory.

It is well known that for an infinitely long bearing the derivative $\partial p / \partial z=0$, because of which the modified Reynolds equation for such kind of bearings is obtained from (22) and written as

$$
\frac{\partial}{\partial x}\left\{\frac{1}{\eta}\left[\frac{h^{3}}{12} \frac{\partial p}{\partial x}+k \frac{h^{5}}{80}\left(\frac{\partial p}{\partial x}\right)^{3}\right]\right\}=\frac{\omega r}{2} \frac{\partial h}{\partial x}
$$

Using (23) and (24) a non-dimensional form of (27) satisfies the equation: 


$$
\frac{\partial}{\partial \theta}\left[\frac{H^{3}}{12} \frac{\partial \Pi}{\partial \theta}+\psi \frac{36 H^{5}}{80}\left(\frac{\partial \Pi}{\partial \theta}\right)^{3}\right]=\frac{1}{12} \frac{\partial H}{\partial \theta} .
$$

The pressure distribution for the case of an infinitely short bearing is governed by the following equation, which is also a reduced form of (22):

$$
\frac{\partial}{\partial z}\left\{\frac{1}{\eta}\left[\frac{h^{3}}{12} \frac{\partial p}{\partial z}+k \frac{h^{5}}{80}\left(\frac{\partial p}{\partial z}\right)^{3}\right]\right\}=\frac{\omega r}{2} \frac{\partial h}{\partial x}
$$

Here the second term on the left hand side of (22) is vanished since $\partial p / \partial x \square$ Dimensionless form of (29) is obtained by using of (23) and (24) and is given as:

$$
\alpha^{2} \frac{\partial}{\partial z_{1}}\left[\frac{H^{3}}{12} \frac{\partial \Pi}{\partial z_{1}}+\alpha^{2} \psi \frac{36 H^{5}}{80}\left(\frac{\partial \Pi}{\partial z_{1}}\right)^{3}\right]=\frac{1}{12} \frac{\partial H}{\partial \theta}
$$

Both of the equations for infinitely long and short bearings, respectively, can be solved by analytical or numerical methods as several solutions of similar type of equations are already published.

\section{Conclusions}

This paper presents precisely, step by step, with necessary explanation obtaining of a modified Reynolds equation for lubrication of finite length journal bearing lubricated with non-Newtonian Rabinowitsch fluid.

Starting from the momentum and continuity equations, and based on the cubic stress constitutive equation, the velocity components for two dimensional flow of a Rabinowitsch fluid are obtained. For this purpose differentiation and integration techniques are applied.

The constitutive equations for the shear stress and shear strain rate are represented by a cubic function of a special type, which finally, after mathematical treatment, are reduced to semi-cubic equations for their components. An approximation to the real root of semi-cubic equation is used such as in [1]. It is possible to find by Cardano's method all roots of the semi-cubic equation, and in further mathematical processing to operate with some appropriate their approximations (due to the complexity of Cardano's formula).

Afterwards, a standard approach for the lubrication theory is applied. Integrating the continuity equation across the fluid film and substituting the obtained velocity components in it, the nonlinear modified 2D Reynolds type equation is derived. The obtained equation is slightly differing in coefficients from those in [1]. This equation is in the appropriate form for numerical modelling and solving by some mesh methods or adapted computer codes ones.

Based on the presented above, a study of the dilatant and pseudoplastic nature of the lubricant in comparison with Newtonian fluid can be carried out. The performances of journal bearings lubricated with a non-Newtonian Rabinowitsch fluid can be compared with the case of Newtonian lubricant through the variation of the non-Newtonian parameter, i.e. the nonlinear factor $\psi$. The future analyses can be performed with combinations of other effects in lubrication performances of journal bearings as influence of fluid inertia, elastic deformations effects, surface roughness, etc. 


\section{References}

1. S. Wada, H. Hayashi, Theoretical and experimental studies, Bulletin of JSME 14, 279286 (1971)

2. C. Rajalingham, B.V. Rao, S. Prabu, Wear 50, 47-57 (1978)

3. P. Bourging, B. Gay, Journal of Tribology 106, 285-290 (1984)

4. S. Sharma, S. Jain, P. Sah, Tribology International 33, 507-517 (2000)

5. J. Javorova, A. Radulescu, R. Lovchalieva, N. Nikolov, P. Kostova, Trib. J Bultrib III, 248-252 (2012)

6. J. Javorova, A. Mazdrakova, I. Andonov, A. Radulescu, Tribology in Industry, 38, 186-196 (2016)

7. P.B. Kushare, C.S. Sharma, Tribology International 69, 84-101 (2014)

8. J. Lin, T. Hung, C. Lin, J of Mechanics, doi.org/10.1017/jmech.2017.48, (2017)

9. H. Hashimoto, S. Wada, J of Tribology 108, 282-288 (1986)

10. J. R. Lin, Tribology International 52, 190-194 (2012)

11. N.B. Naduvinamani, M. Rajashekar, A. Kadadi, Tribol. Int. 73, 78-82 (2014)

12. V. Shigehalli, B. Hanumagowda, C. Rajani, Int J Mathem Arch 8, 36-46 (2017)

13. J.R. Lin, Tribology Letters 10, 237-243 (2001)

14. J. Lin, L. Chu, C. Hung, R. Lu, M. Lin, Z. Naturforsch. 68a, 291-299 (2013)

15. J.R. Lin, Appl Mathem Modelling 38, 392-404 (2014)

16. U.P. Singh, R.S. Gupta, V.K. Kapur, Tribol. Trans. 54, 723-29 (2011)

17. U.P. Singh, R.S. Gupta, V.K. Kapur, IMechE Journal of Engineering Tribology 227, 34-42 (2012)

18. U.P. Singh, R.S. Gupta, V.K. Kapur, Tribology in Industry 34, 128-137 (2012)

19. U.P. Singh, Archive of Mechanical Engineering LX, 247 - 267 (2013)

20. U.P. Singh, Int J Eng Sci Reviews 14(4), 1411-1420 (2014).

21. A. Walicka, E., Walicki, M. Ratajczak, Proc. 4th Minsk Int. Heat Mass Transfer Forum, Minsk Belarus, 19-29 (2000)

22. M. Ratajczak, A. Walicka, E. Walicki, Problems of Machines Exploitation 44, 159-170 (2006)

23. A. Walicka, E., Walicki, Int. J. Appl. Mech. Enging 15, 895-907 (2010)

24. A. Walicka, E. Walicki, P. Jurczak, J. Falicki, Int. J. Applied Mechanics and Engineering 22, 717-737 (2017)

25. A. Cameron, Basic lubrication theory (Chichester, Ellis Horwood, 1976)

26. C. Hung, Education Specialization 97, 87-97 (2009) 